\title{
Topology Adaptive Active Membrane
}

\author{
Sitansu Kumar Das and Dipti Prasad Mukherjee \\ Electronics and Communication Sciences Unit \\ Indian Statistical Institute, Kolkata, India
}

\begin{abstract}
Segmentation of multiple objects especially when the objects are touching each other in an image is a challenging problem. In this paper we propose a parametric active membrane, which can change its topology to detect multiple objects present in the image. The membrane evolves in image space and also along the image intensity surface and if requires, splits into multiple membranes. The methodology is tested for a number of real images that demonstrates the efficacy of the proposed scheme.
\end{abstract}

Keywords: Active membrane, topology adaptive parametric model.

\section{Introduction}

Multiple object detection in a scene using a membrane like surface is always a challenging problem. The topology adaptive parametric active contours [1, [2], 3] and region-based surface fitting approach [4] were used to detect multiple objects in a scene. However, for the conjoint or touching objects sharing common boundary, topology adaptive snakes fail to identify the objects separately. We have designed a dynamic evolving membrane to identify the conjoint objects efficiently.

In our membrane model we assume that the membrane moves from the top of the image surface to approximate the image intensity such that the sum of internal energy and external energy of the approximated membrane is minimum. The membrane internal energy is responsible for maintaining the smoothness of the approximated surface while the image intensity influenced external energy drives the membrane towards the image surface. The force governing membrane evolution is applied along the image plane. Once the deformation of membrane exceeds the allowable deformation limit that membrane can sustain, tearing of membrane occurs in the high-stressed zone and then the membrane pieces continue propagation once again. The membrane fitting process continues until the deformation process of membrane comes to equilibrium when internal and external energy of the membrane balance each other. The membrane at the equilibrium state defines multiple objects present in the image.

The membrane is modeled using finite element method [5], 6] and the partial differential stress equation is solved at the finite element vertices [7, 8], 3]. In [1] topology adaptive active contour is used where the image surface is tessellated using triangular elements and the splitting and joining of parametric active 
contour(s) depends on the sign of vertices at the triangular element. This is similar to the signed distance function based level set approach [9] and computationally expensive. Moreover, the approach in 1 1 only relies on edge force and therefore, cannot segment the conjoint objects. Conjoint objects are distinguishable from one another because each of them is defined by a specific band of intensities compared to their adjoining neighborhood objects. Our dynamic membrane approach segments the image within different bands of intensities and therefore can segment conjoint objects efficiently. This is one of the specific contributions of our proposed work. We have presented the result and compared our approach with [1] in Section 3 followed by conclusion. But before that in the next section we present our model of active membrane and its evolution.

\section{Methodology}

For the proposed 3D membrane we consider an image $I(x, y): \Re \times \Re \rightarrow \Re$ as a $3 \mathrm{D}$ surface where an image pixel value is the height of the surface along $z$-axis at an image location $(x, y)$. Different types of the undulations are viewed on the image surface due to intensity difference created by the presence of objects in the image.

Simulation of membrane for image segmentation consists of two major parts. The first part deals with driving the membrane toward the image surface. In the second part the elongation of the membrane due to the deformation constrained by the image characteristics is calculated and tearing of membrane is carried out depending on the stress map of the membrane. While first part requires solution of PDE for the membrane, in the second part calculated stress is compared with preset value. In the next section we formulate the construction and evolution of membrane model.

\subsection{Design of Membrane}

We define the membrane as $v(r, s, t)$, where $r$ and $s$ are $2 \mathrm{D}$ co-ordinates on the membrane surface and $t$ is the time parameter for membrane evolution. The membrane proceeds towards the image surface by minimizing the energy functional,

$$
E(v)=\iint\left[\alpha(I)\left(\left\|\frac{\partial v}{\partial r}\right\|^{2}+\left\|\frac{\partial v}{\partial s}\right\|^{2}\right)+\beta(I) P(I)\right] d r d s
$$

In (11) the first two terms in the right hand side are the internal energy terms dependent on the amount of deformation of the membrane. The last term is an external energy term dependent on the image characteristics. The weights for the internal and external energies are $\alpha(I)$ and $\beta(I)$ respectively which depends on the image property. The external energy $P(I)$ depends on the image gradient and also on the difference of local heights of membrane and image intensity surface. Minimization of $E(v)$ is done by

$$
\frac{\partial v}{\partial t}+\frac{\partial E(v)}{\partial v}=0
$$


where the evolution of the membrane $v$ has to be carried out in $3 \mathrm{D}$ both in image space $(x, y)$ and along intensity axis $z$. Next we present the numerical implementation scheme of (2).

\subsection{Numerical Implementation}

Solving (2) for the membrane needs the assumption of the existence of an imaginary grid over the membrane. The grid tessellates the membrane into a mesh of small triangular elements. The grid points over the membrane are represented in a matrix form referred as grid matrix. If the membrane is tessellated into a mesh of $2 m n$ triangular elements, then there exist $m+1$ rows and $n+1$ columns in the grid matrix. One typical element of the membrane is denoted by $e$ and we assume that we know the properties of the surface in the three vertices of $e$ [7, 8]. We use linear basis function using weighted combinations of the element vertices since only first order PDE is used to evolve the membrane. Following 6] the basis function is given by,

$$
p_{e}=a r+b s+c, a=\frac{1}{h}\left(v_{2,1}-v_{1,1}\right), b=\frac{1}{h}\left(v_{1,2}-v_{1,1}\right), c=v_{1,1}
$$

where $v_{1,1}, v_{1,2}$ and $v_{2,1}$ are the vertices of the element as shown in the Fig. 1 Discrete version of (11) using (3) is given by,

$$
\begin{aligned}
E(V)= & \frac{h^{2}}{2} \sum_{i=1}^{m+1} \sum_{j=1}^{n+1} \alpha\left(I\left(x_{i, j}, y_{i, j}\right)\right)\left[\left(v_{i+1, j}-v_{i, j}\right)^{2}+\left(v_{i, j+1}-v_{i, j}\right)^{2}\right] \\
& +\beta\left(I\left(x_{i, j}, y_{i, j}\right)\right) P\left(I\left(x_{i, j}, y_{i, j}\right)\right)
\end{aligned}
$$

where $\left(x_{i, j}, y_{i, j}\right)$ is the image location at $(i, j)$ th grid point. The discrete vertices of the elements are $V=\left(v_{1,1}, v_{2,1}, \ldots, v_{i, j}, \ldots, v_{m+1, n+1}\right)$ and $v_{i, j}=\left(x_{i, j}, y_{i, j}, z_{i, j}\right)$

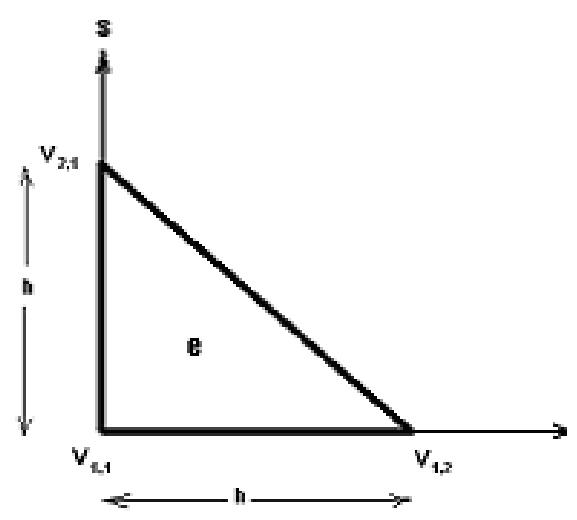

Fig. 1. A triangular finite element 
denotes the co-ordinate of the $(i, j)$ th grid point. Differentiating $E(V)$ with respect to $V$ implies differentiating (4) with respect to $v_{i, j}$ for $i=1, \ldots, m+1$ and $j=1, \ldots, n+1$ and we get $(m+1)(n+1)$ number of equations. However these equations are nonlinear due to the dependability of $\alpha$ and $\beta$ on $I\left(x_{i, j}, y_{i, j}\right)$. Therefore, to facilitate linear solution, we assume $\alpha$ and $\beta$ are constants in each iteration and they are obtained from the membrane at previous iteration. So differentiating (4) with respect to $v_{i, j}$ gives,

$$
\begin{aligned}
& \alpha\left(I\left(x_{i, j}^{t-1}, y_{i, j}^{t-1}\right)\right)\left(4 v_{i, j}^{t}-v_{i-1, j}^{t}-v_{i+1, j}^{t}-v_{i, j-1}^{t}-v_{i, j+1}^{t}\right) \\
& +\beta\left(I\left(x_{i, j}^{t-1}, y_{i, j}^{t-1}\right)\right)\left(\frac{\partial P\left(I\left(x_{i, j}^{t-1}, y_{i, j}^{t-1}\right)\right)}{\partial x}, \frac{\partial P\left(I\left(x_{i, j}^{t-1}, y_{i, j}^{t-1}\right)\right)}{\partial y}, \frac{\partial P\left(I\left(x_{i, j}^{t-1}, y_{i, j}^{t-1}\right)\right)}{\partial z}\right)=0
\end{aligned}
$$

Three discrete equations can be obtained from (15),

$$
\begin{aligned}
& \alpha\left(I\left(x_{i, j}^{t-1}, y_{i, j}^{t-1}\right)\right)\left(4 x_{i, j}^{t}-x_{i-1, j}^{t}-x_{i+1, j}^{t}-x_{i, j-1}^{t}-x_{i, j+1}^{t}\right)=\beta\left(I\left(x_{i, j}^{t-1}, y_{i, j}^{t-1}\right)\right) \frac{\Delta P}{\triangle x} \\
& \alpha\left(I\left(x_{i, j}^{t-1}, y_{i, j}^{t-1}\right)\right)\left(4 y_{i, j}^{t}-y_{i-1, j}^{t}-y_{i+1, j}^{t}-y_{i, j-1}^{t}-y_{i, j+1}^{t}\right)=\beta\left(I\left(x_{i, j}^{t-1}, y_{i, j}^{t-1}\right)\right) \frac{\triangle P}{\triangle y} \\
& \alpha\left(I\left(x_{i, j}^{t-1}, y_{i, j}^{t-1}\right)\right)\left(4 z_{i, j}^{t}-z_{i-1, j}^{t}-z_{i+1, j}^{t}-z_{i, j-1}^{t}-z_{i, j+1}^{t}\right)=\beta\left(I\left(x_{i, j}^{t-1}, y_{i, j}^{t-1}\right)\right) \frac{\triangle P}{\Delta z}
\end{aligned}
$$

The external force component in the image plane is Gaussian convolved image gradients $|\nabla G(I)|$. Given the weight of the external force $N(k)$ at the $k$ th point out of total $q$ number of discrete points in the inter-vertex distance, $N(k)=$ $\left(1-\frac{k}{q}\right), k=0, \ldots, q$, the external force component is given by,

$$
\begin{aligned}
& \left(\frac{\triangle P}{\Delta x}\right)_{i, j}=\sum_{\begin{array}{l}
(\xi, \eta)=v_{i+1, j}, v_{i-1, j} \\
v_{i, j+1}, v_{i, j-1}
\end{array}} \sum_{k=0}^{q} N(k)\left|\nabla_{x} G\left(I\left(x_{i, j}+\frac{x_{i, j}-\xi}{q} k, y_{i, j}+\frac{y_{i, j}-\eta}{q} k\right)\right)\right| \\
& \left(\frac{\triangle P}{\triangle y}\right)_{i, j}=\sum_{\substack{(\xi, \eta)=v_{i+1, j}, v_{i-1, j} \\
v_{i, j+1}, v_{i, j-1}}} \sum_{k=0}^{q} N(k)\left|\nabla_{y} G\left(I\left(x_{i, j}+\frac{x_{i, j}-\xi}{q} k, y_{i, j}+\frac{y_{i, j}-\eta}{q} k\right)\right)\right|
\end{aligned}
$$

The derivative of the external force in the $z$-direction essentially depends on the difference of membrane height and the image intensity. The force to drive the membrane towards image surface minimizes $\rho=\left(I\left(x_{i, j}, y_{i, j}\right)-z_{i, j}\right)$. Therefore, we model $\left(\frac{\triangle P}{\triangle Z}\right)_{i, j}=-\rho(1+\exp (|\rho|))$. The weights $\alpha(I)$ (and similarly $\left.\beta(I)\right)$ are taken as linear function $\alpha(I(x, y))=\alpha_{\text {low }}+\frac{I(x, y)}{255} \times\left(\alpha_{\text {high }}-\alpha_{\text {low }}\right)$ where $\left(\alpha_{\text {low }}, \alpha_{\text {high }}\right)$ are set experimentally.

Equation (6) can be rewritten as $\alpha \cdot * A X=\beta \cdot * F x$, and $\alpha \cdot * A Y=\beta \cdot * F y$, $\alpha \cdot * A Z=\beta \cdot * F z$ where $A$ is the $(m+1)(n+1) \times(m+1)(n+1)$ pentadiagonal matrix referred as the stiffness matrix. The position vectors of the element vertices are $X, Y$ and $Z$ and $F x, F y$ and $F z$ are the force vectors at the membrane node points. The operation ' $*$ ' denotes element wise multiplication. We assume that we have a priori estimation $X^{t-1}, Y^{t-1}$ and $Z^{t-1}$ at iteration $(t-1)$ for 
the current iteration is $t$. Then, the final discrete form of (2) for the evolving membrane is given by,

$$
\begin{aligned}
& \frac{X^{t}-X^{t-1}}{\triangle t}+\alpha \cdot * A X^{t}=\beta \cdot * F x \Rightarrow(I+\triangle t \times \alpha \cdot * A) X^{t-1}=\left(X^{t-1}+\triangle t \times \beta \cdot * F x\right) \\
& \frac{Y^{t}-Y^{t-1}}{\triangle t}+\alpha \cdot * A Y^{t}=\beta \cdot * F y \Rightarrow(I+\triangle t \times \alpha \cdot * A) Y^{t-1}=\left(Y^{t-1}+\triangle t \times \beta \cdot * F y\right)(8) \\
& \frac{Z^{t}-Z^{t-1}}{\triangle t}+\alpha \cdot * A Z^{t}=\beta \cdot * F z \Rightarrow(I+\triangle t \times \alpha \cdot * A) Z^{t-1}=\left(Z^{t-1}+\triangle t \times \beta \cdot * F z\right)
\end{aligned}
$$

where $I$ and $\triangle t$ denotes identity matrix and time step respectively. Next we describe the tear model based on which the membrane splits into pieces.

\subsection{Tear Model}

Tearing process involves deletion of connectivity between two neighbouring vertices. A vertex may have maximum four and minimum zero connected neighbours, and in the later case the vertex is deleted. As a result the stiffness matrix $A$ is changed due to the vertex deletion. If $(i, j)$ th vertex is connected to its $(k, l)$ th neighbour and the connectivity is lost due to the deletion process then $R_{i, j}$ and $R_{k, l}$ rows of $A$ is modified where $R_{i, j}=(i-1)(n+1)+j$ and $R_{k, l}=(k-1)(n+1)+l$. In $A$ the diagonal elements of $R_{i, j}$ and $R_{k, l}$ rows is reduced by one and $\left(R_{i, j}, R_{k, l}\right)$ and $\left(R_{k, l}, R_{i, j}\right)$ elements are changed to zero. If $(i, j)$ th vertex of the grid matrix is deleted then the row and column corresponding to $R_{i, j}$ is deleted and $A$ is reduced in size by one both in row and column. Deletion of a connection deletes the two elements sharing the connection. Also, deletion of two elements in the two sides of a connection also deletes the connection. The criterion to delete a connection is the excessive elongation of the element governed by (8). When the elongation of a connection exceeds a preset distance it is disconnected. We keep this distance variable and make it dependent on the variation of local intensity. Taking preset distance as function of image intensity allows us to take variable distance for tearing the membrane in the different parts of image. We take the distance function as

$$
d(x, y)=d_{\text {low }}+\frac{I(x, y)}{255} \times\left(d_{\text {high }}-d_{\text {low }}\right)
$$

where, $d_{\text {high }}$ and $d_{\text {low }}$ are application dependent preset constants.

So for each iteration we compare the inter vertex Euclidean distance between a pair of neighbouring nodes with its preset distance $d(x, y)$ and also for each connected link we check the existence of its two neighbourhood elements. If inter vertex distance exceeds preset limit or there exists no neighbourhood element in either side of the connection then connectivity between the vertices is deleted. After this we delete all the vertices having zero connected neighbour. Simultaneously, as mentioned earlier, we modify $A$ deleting corresponding row column and start the next iteration. In the next section we discuss the results of our proposed method. 


\section{$3 \quad$ Results}

We have implemented the proposed methodology to detect objects in a wide variety of real images, especially on some low-contrast images where multiple objects are present. But, first a set of examples to show that it works equally well for simple image segmentation problem.

For the examples of Figs. 2 and 3, the active membrane is evolved following (8). For poorly contrasted white blood cells, segmentation of cells having different shapes can be achieved from a single initialization of the membrane. In all the

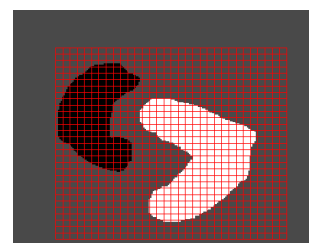

(a)

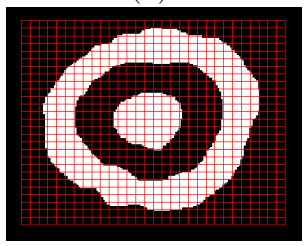

(d)

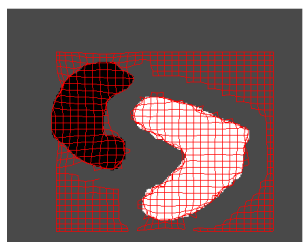

(b)

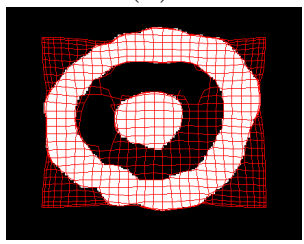

(e)

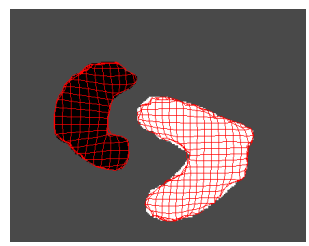

(c)

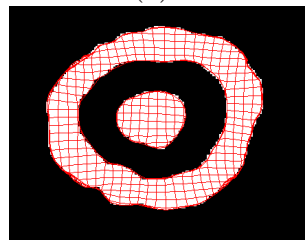

(f)

Fig. 2. (a), (d): Initial membrane on synthetic objects. (b), (e): Intermediate stages of membrane evolution after 20 iterations. (c), (f): Final segmentation using topology adaptive membrane after 150 iterations.

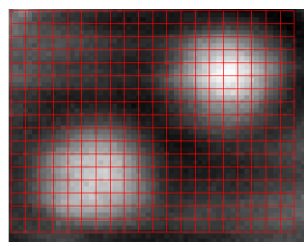

(a)

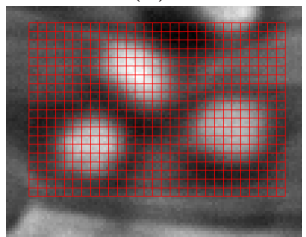

(d)

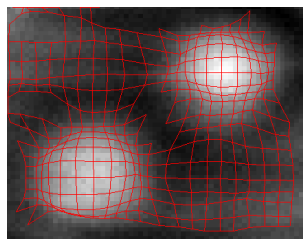

(b)

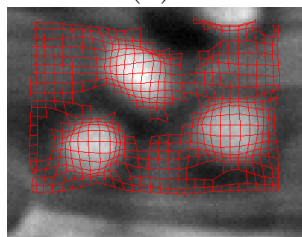

(e)

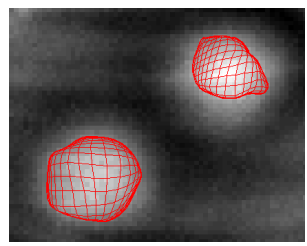

(c)

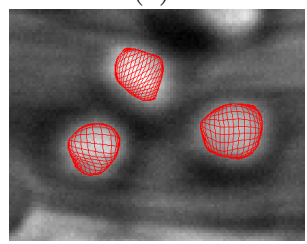

(f)

Fig. 3. (a), (d): Multiple white blood cells with initial active membrane. (b), (e): After 15 iterations of the membrane. (c), (f): After segmentation using 180 iterations. 
above examples, we take $d_{\text {high }}=5 g_{d}$ and $d_{\text {low }}=1.1 g_{d}$ where $g_{d}$ is the grid distance and $\alpha_{\text {high }}=0.05, \alpha_{\text {low }}=0.03, \beta_{\text {high }}=0.001$ and $\beta_{\text {low }}=0.01$. The entire approach was implemented in Matlab 6.5 in Pentium 4, $2.1 \mathrm{GHz}$ PC. In the next section we compare our method with topology adaptive snake 1].

\subsection{Comparison}

Proposed method has two advantages over topology adaptive contour [1]. The first advantage is that the proposed approach uses automatic initialization, where as for 11 separate initialization is required for a group of objects. The second advantage is that the proposed approach can detect touching objects sharing a common boundary as it looks for both image gradients and image intensity surface as opposed to only image gradient information in 1 .

The touching objects of Fig. 4(a) share common boundary with an initial membrane placed on the image. Fig. 4(b) shows membrane detects the two objects correctly and separately. The results of Fig. 4(c) and (d) show that the approach in [1] fails for this kind of situation. For Fig. 44(c) the initial contour is outside the object while the same for Fig. 4(d) is inside the object. The active contour of [1] was contracting in case of Fig. 4(c) while it was expanding in case of Fig. 4(d). In either case, the topology adaptive snake fails to capture conjoint object correctly and separately, which can be achieved using the proposed method.

To compare the performance of our method numerically we define a performance measure coefficient as $P M C=1 /\left(1+\sum_{i=1}^{n} d_{i}\right)$, where $n$ is the number of contour points and $d_{i}$ is the distance between the $i$ th contour point to its closest object boundary point. Therefore, the closer the value of $P M C$ to 1 the better is the segmentation. Table 1 shows that as expected, the proposed approach performs much better than the topology adaptive contour [1].

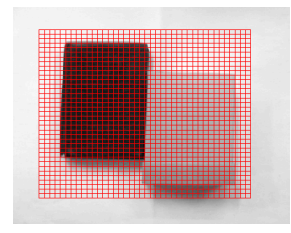

(a)

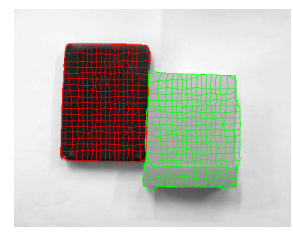

(b)

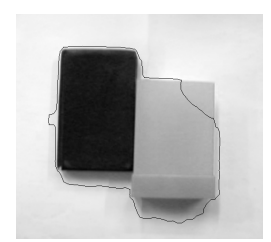

(c)

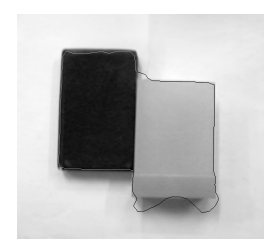

(d)

Fig. 4. (a): Initial membrane on the conjoint objects. (b) After correct segmentation of the touching objects after 200 iterations. (c)-(d) Topology adaptive active contour evolution 1 for different initialization after 250 iterations.

Table 1. PMC of the proposed approach and topology adaptive contour [1]

\begin{tabular}{|c|c|c|c|}
\hline & Synthetic image & Blood cell image & Conjoint object image \\
\hline Proposed approach & 0.0109 & 0.0044 & 0.0011 \\
\hline Topology adaptive contour [1] & 0.0041 & 0.00031 & 0.000017 \\
\hline
\end{tabular}




\section{Conclusions}

We have developed a topology adaptive membrane to segment multiple conjoint objects. The proposed approach is initialization independent where an initial membrane without any a priori knowledge of the objects evolves and adapts to the shape of objects present in the image. Comparison of the proposal with similar approach shows promise. Our goal is to use this technique in a tracking framework constraining the membrane evolution with shape and motion information in a video.

\section{References}

1. McInerney, T., Terzopoulous, D.: T-snake: Topology adaptive snakes. Medical Image Analysis 4, 73-91 (2000)

2. Kass, M., Witkin, A., Terzopoulous, D.: Snakes: Active contour models. Int. J. Computer Vision 1(4), 321-331 (1988)

3. Cohen, L., Cohen, I.: Finite-element methods for active contour models and balloons for 2-d and 3-d images. IEEE Trans. Pattern Analysis Machine Intelligence 15(11), 1131-1147 (1993)

4. Besl, P., Jain, R.: Segmentation through variable order surface fitting. IEEE Trans. Pattern Analysis Machine Intelligence 10(2), 167-192 (1988)

5. McInerney, T., Terzopoulous, D.: Non-rigid motion tracking. In: IEEE proceedings, vol. 4, pp. 73-91 (2000)

6. Terzopoulous, D.: The computation of visible-surface representations. IEEE Trans. Pattern Analysis Machine Intelligence 10(4), 417-438 (1988)

7. Desai, C.: Elementary Finite Element Method. Prentice-Hall, Englewood Cliffs (1979)

8. Hinton, E., Owen, D.: An Introduction To Finite Element Computations. Pineridge Press Limited (Swansea) (1981)

9. Mukherjee, D., Ray, N., Acton, S.: Level set analysis for cell detection and tracking. IEEE Trans. on Image Processing 13(4), 562-572 (2004) 